\title{
Mixing Through Stirring of Steady Flow in Small Amplitude Helical Tubes
}

\author{
A. N. Cookson, D. J. Doorly, S. J. Sherwin \\ Department of Aeronautics, Imperial College London, South Kensington, London, \\ $S W 72 A Z, U K$
}

\begin{abstract}
In this paper we numerically simulate flow in a helical tube for physiological conditions using a co-ordinate mapping of the Navier-Stokes equations. Helical geometries have been proposed for use as bypass grafts, arterial stents and as an idealised model for the out-of-plane curvature of arteries. Small amplitude helical tubes are also currently being investigated for possible application as A-V shunts, where preliminary in vivo tests suggest a possibly lower risk of thrombotic occlusion. In-plane mixing induced by the geometry is hypothesised to be an important mechanism. In this work, we focus mainly on a Reynolds number of 250 and investigate both the flow structure and the in-plane mixing in helical geometries with fixed pitch of 6 tube diameters (D), and centreline helical radius ranging from $0.1 D$ to $0.5 D$. High-order particle tracking, and an information entropy measure is used to analyse the in-plane mixing. A combination of translational and rotational reference frames are shown to explain the apparent discrepancy between flow field and particle trajectories, whereby particle paths display a pattern characteristic of a double vortex, though the flow field reveals only a single dominant vortex. A radius of $0.25 D$ is found to provide the best trade-off between mixing and pressure loss, with little increase in mixing above $R=0.25 D$, whereas pressure continues to increase linearly.
\end{abstract}


Key words: laminar, spectral/hp, graft, shunt, stent, pipe, thrombosis, co-ordinate mapping, advection

\section{Introduction}

Almost 30,000 coronary artery bypass graft procedures are performed each year in the UK according to the British Heart Foundation, however over 50\% of CABG fail within 10 years due to the development of neo-intimal hyperplasia (Bryan and Angelini, 1994). Similarly, arterio-venous shunts constructed from ePTFE are prone to occlusion by thrombosis and intimal hyperplasia. In the United States alone there are 175,000 ePTFE grafts used for permanent vascular access, with the 1 and 2-year primary patency rates currently at $50 \%$ and $25 \%$ respectively. Consequently, much research has been conducted in the past few decades to design grafts that will remain patent for far longer, ideally longer than the life-span of the patient.

A promising avenue of this research, initiated by Caro et al. (Caro, C. G. et al., 1996) (Sherwin et al., 2000), is to use out-of-plane geometries that induce fully three-dimensional, physiologically-realistic swirling flows, and produce more uniform wall shear stress distributions. However, in a clinical environment, such geometries cannot be guaranteed to be preserved after wound closure. Greater control of geometry is possible with vascular prostheses, with small amplitude helical tubes being proposed (Caro et al., 2005). The helical geometry induces the necessary swirling flow, whilst also being mechanically robust, and has undergone preliminary in-vivo trials, and subsequently a preliminary clinical study Huijbregts et al. (2007). Caro et al. hypothesised that the inplane mixing induced by the helical geometry and the more uniform WSS 
distribution are responsible for preventing graft occlusion from thrombosis and neo-intimal hyperplasia. Likewise a new design of arterial stent has been proposed, which when inserted into the host artery and expanded, enforces a helical tube boundary at the artery wall. This is an alternative procedure to the helical bypass graft, but the operating conditions, e.g. Reynolds number, will be comparable to those of a bypass graft.

It is to be emphasized that the benefits of helical geometry in vascular conduits have yet to be firmly established, although they appear promising. The range of possible configurations is large, and how the haemodynamics responds to changes in geometric parameters has not been studied in detail. Systematic investigation of the effects of helical geometry on the haemodynamics are needed, not only to inform potential designs of prostheses and surgical vascular reconstructions, but to improve our understanding of the normal vasculature. As pointed out by Zabielski and Mestel (1998), a helical pipe serves as an idealization of many arterial geometries. The mechanisms governing mixing in such geometries may provide insight into cardiovascular diseases such as atherosclerosis, which are thought to be linked to wall shear stress and wall transport. These considerations provide the motivation for the work described below, in which a range of small amplitude helical geometries, representative of possible prosthetic vascular configurations or native tortuous arteries are the subject of studies of flow structure and mixing. 


\section{Methods}

\subsection{Parameters of Study}

A helix is a three-dimensional space curve that can be described by the equations:

$$
x=R \cos (z / c), \quad y=R \sin (z / c)
$$

where $R$ is the radius or amplitude of the helix and $c$ is a constant parameter, such that the wavelength or one pitch of the helix equals $2 \pi c$. For a helical tube the internal radius is an additional parameter $D / 2$, as illustrated in Figure 1. The Frenet triad, which consists of the normal, N, binormal, B, and tangent $\mathbf{T}$, vectors, is often used to define a co-ordinate system along a curve. A feature of helical flows is that the velocity field is self-similar along the axis, and therefore the entire field can be represented by a single cross-section normal to the centreline, which rotates with the Frenet triad along the curve. This property is illustrated in Figure 2, for a helical geometry with $R=0.25$ and pitch length $6 D$. Contour plots of the axial velocity, $w$, are shown at $1 / 4$, $1 / 2$ and $3 / 4$ of the pitch, and demonstrate both the self-similarity and rotation of the flow field. The location of the axis origin $(x=0=y)$ is marked by a cross on each contour plot.

A Reynolds number of 250 , defined as $\frac{\bar{w} D}{\nu}$ is used for the majority of the flow studies to match representative physiological conditions in a bypass graft, as used in other studies (Sherwin et al., 2000), and is also appropriate for stent applications and as a model for flow in larger arteries. For the case of an A-V shunt, the Reynolds number is several times larger, in the range 900- 


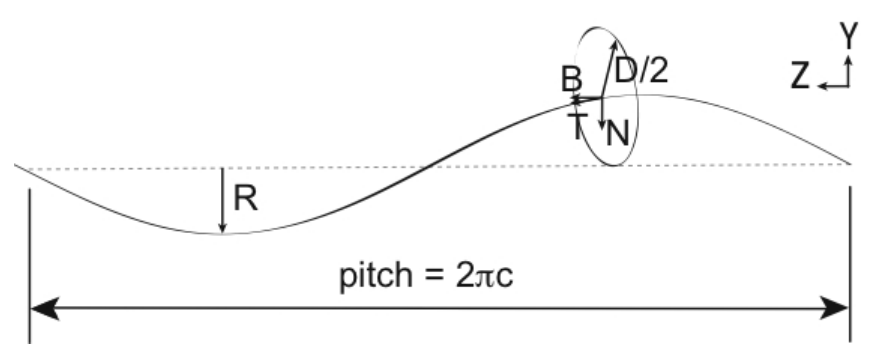

Fig. 1. Geometric parameters of a helix

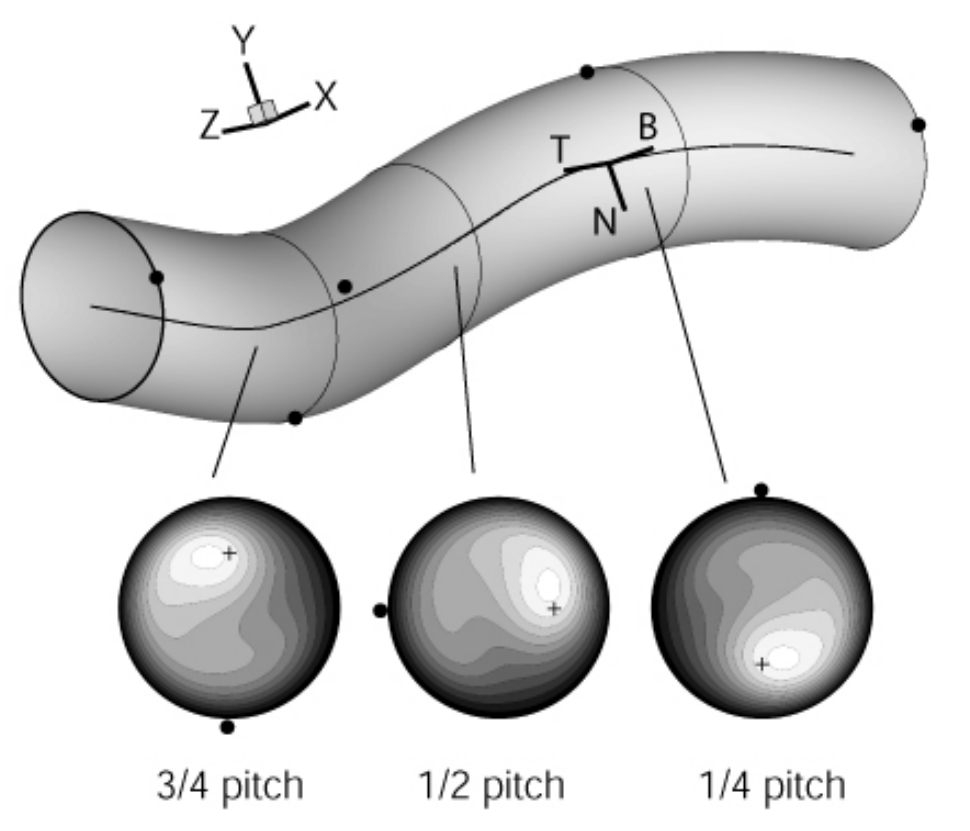

Fig. 2. Helical tube geometry and self similar velocity field

1800 (Loth et al., 2008). To provide some indication of the flow dynamics and mixing behaviour at these Reynolds numbers, some computations were also performed in the range 500-700.

Caro et al. coin the term, small amplitude helical technology (SMAHT), to describe the geometries used for clinical applications, which have small helical radius, but large pitch length. The upper limit of the helical pitch length that can be used for SMAHT is set by the physical space constraints of the clinical application, with the lower limit determined by the necessity to preserve the general morphology of the SMAHT. Within these limits it is expected that 
the flow field will undergo only small changes with respect to varying pitch, relative to those induced by varying the radius. Therefore in this study, and taking the geometries used by Caro et al. as a guide, all the helical geometries will have a pitch of six tube diameters, $6 D$, with the helical radius varying from $0.1 D$ to $0.5 D$ in increments of $0.05 D$.

In the following work we assume blood to be a Newtonian fluid, which is a reasonable approximation for flow in the larger blood vessels (Friedman, 1993). Only steady flow is examined at present, which for modelling A-V shunt flow is acceptable, as relatively low pulsatility has been found in renal dialysis access shunt flow (Caro et al., 2005). For flow in artery bypass grafts and stents, it is a poorer approximation, but a significant portion of the pulsatile flow cycle is quasi-steady, so that the vortical flow structures may be similar, though as recognised in (Doorly, 1999), unsteadiness can play a large role in vascular mixing.

\subsection{Numerical scheme}

Various approaches to solving the flow in a helical tube have been taken in the literature, starting with Wang's non-orthogonal co-ordinate system (Wang, 1981), which used the Frenet triad as the co-ordinate axes. This was followed by Germano's orthogonal refinement (Germano, 1981) of Wang's system, to the more recent and computationally efficient helically symmetric co-ordinate system introduced by Zabielski and Mestel (1998). The current body of research into helical tube flows can be crudely summarised by remarking that, at the relatively modest Reynolds numbers we consider, curvature creates a two-vortex solution, in the manner of a Dean flow, with an asymmetric axial 
velocity profile. The effect of torsion is to rotate this flow profile along the centreline, as shown in Figure 2, and to distort the vortical structure. This leads to one vortex dominating over the other, and for certain parameter ranges the second vortex is effectively eliminated.

The approach taken here to obtain the velocity field is different from those of previous researchers, and is only valid for use on helical tubes with small radius $R$. For these geometries a helical tube can be reasonably approximated by a circular cross-section translated by a helical centreline. Conceptually, reversing this approximation implies that a co-ordinate transformation can be applied to the Navier-Stokes equations in a helical domain, and an existing spectral/hp element code Nektar (Sherwin and Karnaidakis, 2005) used to solve modified Navier-Stokes equations in a cylindrical domain. This permits a Fourier expansion basis in the periodic direction, and therefore a single twodimensional computational mesh can be used for all geometries. This removes the computationally expensive requirement for remeshing different 3-D geometries, in addition to being inherently faster to solve the velocity field than for a true 3-D mesh. This approach has been successfully used to investigate the flow around bluff bodies (Newman, 1996), (Evangelinos, 1999), (Darekar and Sherwin, 2001), and in 3-D channel flows (Koberg, 2008).

\subsection{Particle tracking}

Caro et al.'s preliminary study used labelled particle maps and simple dye injection experiments to demonstrate that helical tubes can cause rapid inplane mixing. Yamamoto et al. allude to mixing in a helical tube, through their use of experimental flow visualisation, and numerical particle tracking 
(Yamamoto et al., 2002), however neither study comprehensively investigated the flow from this perspective or attempted to quantify the mixing behaviour.

As a physical process, mixing is a combination of advection and diffusion. Advection, by stretching and folding the flow, creates large concentration gradients across which species can rapidly diffuse. Previous research has found that for bypass graft flows it is sufficient to consider advection alone, as adding a diffusion model makes little difference to the results, except perhaps in areas of the velocity field where there is recirculating flow (Doorly et al., 2002). Therefore rather than solving the advection-diffusion equation, we need only to integrate the advection equations, shown in Equation (1), with respect to time. Strictly, mixing where only advection is considered should be termed 'stirring'.

$$
\frac{d \mathbf{x}}{d t}=\mathbf{u}(\mathbf{x}, t)
$$

The numerical integration of Equation (1) is accomplished by tracking massless computational particles through the velocity field. The algorithm used is a 4-stage Runge-Kutta time integration scheme, which interpolates directly from the high-order polynomial representation of the velocity field, and is thus more accurate than a scheme using a linear interpolation mesh (Coppola et al., 2001).

\subsection{Entropic measure of mixing}

Conceptually, mixing is the reduction of non-uniformity, or equivalently, the increase of disorder. Information entropy was first defined by Shannon (1948), and can be interpreted as a measure of disorder, naturally leading to its use in 
such diverse fields as mixing in polymer processing (Wang et al., 2003), (Wang et al., 2005b), (Wang et al., 2005a), chaotic micromixers (Kang and Kwon, 2004) and aerosol mixing in the lung (Butler and Tsuda, 1997). Here the formulation used is that introduced by Kang and Kwon (2004). They tracked passive, coloured particles through a chaotic micromixer, taking sections of the trajectories at areas of interest, and having superimposed a grid onto the section, applied Equation (2).

$$
S=\sum_{i=1}^{N_{c}}\left[w_{i} \sum_{k=1}^{N_{s}}\left(n_{i, k} \log n_{i, k}\right)\right]
$$

In Equation (2), $i$ is the cell index, $k$ is the species index, $w_{i}$ is the weighting factor for each cell, $N_{c}$ the number of cells, $N_{s}$ the number of species (i.e. different colours of particles) and $n_{i, k}$ is the particle number fraction of the $k^{\text {th }}$ species in the $i^{\text {th }}$ cell. The weighting factor $w_{i}$ is defined so that it is zero if a cell contains no particles, or only particles of a single species/colour; within such a cell the particle distribution is uniform, and therefore should contribute zero to the entropy summation, i.e. disorder can only occur if particles of different colours are present. The box counting concept is illustrated in Figure 3 , using a reduced number of boxes, along with the corresponding terms used in Equation (3). In Figure 3 particles of only two colours are considered and this is used throughout this work.

As a value considered in isolation, the entropy calculated in (2) has little meaning. Again following Kang and Kwon we define a relative entropy measure $\kappa$, which quantifies the increase in entropy of the particle distribution at a particular cross-section from that of the inlet distribution of particles. This is then normalised by the maximum possible entropy increase from the inlet 


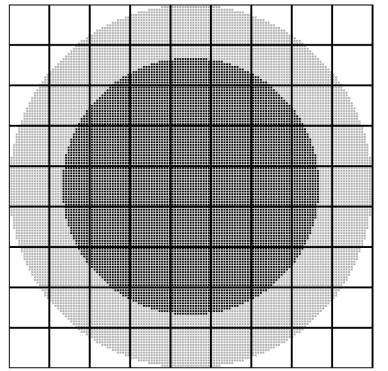

(a) $S_{0}$ : Initial distri-

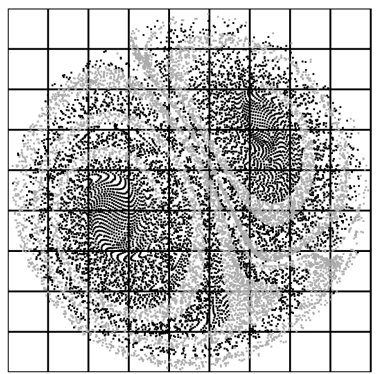

(b) $S: R=0.25 D$ at

$z=30 D$

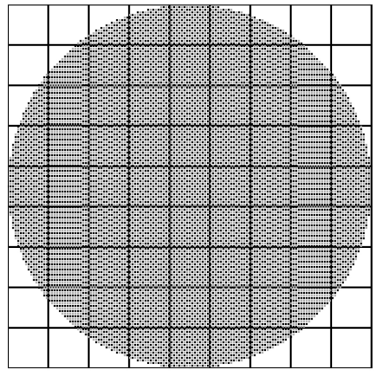

(c) $S_{\max }: \quad$ Perfect mixing

bution

Fig. 3. Particle distributions overlaid with illustrative grid used for entropy calculation

particle distribution, and is defined in Equation (3).

$$
\kappa=\frac{S-S_{0}}{S_{\max }-S_{0}}
$$

From the above it can be appreciated that when $\kappa$ is equal to zero, no mixing has occurred, and when kappa equals one, the mixing is maximised.

This method of characterising mixing relies on two levels of statistical sampling. The first is the seeding of the flow with particles, which will determine the quality of the resolution of the flow features. The second is the box-counting used to calculate the information entropy. Care must therefore be taken both with the number of particles, and the number of boxes, specifically, $N_{\text {particles }} / N_{\text {species }} \gg N_{\text {boxes }} \gg 1$.

Even if this condition is satisfied, it should be noted that the entropy of a given particle distribution will decrease as the number of boxes increases. In fact as $N_{\text {boxes }} \rightarrow \infty$, entropy $S$ will disappear to zero; that is, entropy only exists by virtue of the coarse-graining process of box-counting. This property means 
that it is not straightforward to compare the entropy values calculated for different applications unless the number of particles and boxes are identical.

In Equation (2), the definition of entropy is such that only boxes that contain particles of different colours contribute to the entropy summation. This implies that if a sufficient number of boxes are used, a box containing more than one colour will only do so along the boundary of the interface that divides the colours. In other words, with appropriate resolution, the entropy calculation is analogous to determining the length of the interface that separates the particle species. This point was made in relation to the intensity of segregation measure by Khakhar (1986), but holds equally well for information entropy, as both measures utilise box-counting. This suggests that one way to validate the entropic measure is to use particle distributions with known, or easily determined, interface lengths, and compare the entropy value with the interface length. Unfortunately, as Krasnopolskaya et al. (1999) notes, the measure cannot, in general, be validated this way for an arbitrary number of boxes. However, it is certainly possible to validate the measure for a limited range of particle distributions, which is the approach taken here.

Tests were performed with progressively increasing numbers of concentric rings of particles of different colours. The total interface length of the rings increases linearly with the number of rings. The test cases showed that for roughly 60000 particles, 10000 boxes are needed for the entropy calculation, so that the variation in entropy is also linear. Calculations were performed for several cases using 61527 particles, to validate the data generated using 15371 particles. 


\section{Results and discussion}

\subsection{Variation of Velocity Field with Helical Geometry}

Before examining the results for the velocity field, it should be noted that, contrary to the case of curved tubes, the curvature of all the geometries considered here increases with helical radius. The curvature of a helix is defined as:

$$
\kappa=\frac{R}{R^{2}+c^{2}}
$$

This function is such that for all cases where $R<c$, the curvature will increase with radius. The maximum curvature occurs for $R=c$, beyond which curvature decreases with radius.

As stated earlier, nine different geometries are examined, with helical radius increasing from $0.1 \mathrm{D}$ to $0.5 \mathrm{D}$ in increments of $0.05 \mathrm{D}$, however since the patterns of velocity are similar we only show plots of the velocity field for the cases $R=0.1 D, R=0.3 D$ and $R=0.5 D$.

As outlined earlier, there a several different coordinate systems that can be used to describe the flow in a helical tube. If a helical coordinate system is used, either that of Wang, Germano or Mestel \& Zabielski, then the velocity field in a cross-section is symmetric throughout the tube, that is, a single crosssection will represent the flow in the whole tube. We choose to display both the velocity field and mixing data in a Cartesian co-ordinate system, as this is more convenient for investigating the relationship between the flow and the resultant mixing behaviour. Nonetheless processing the results using Wang's 


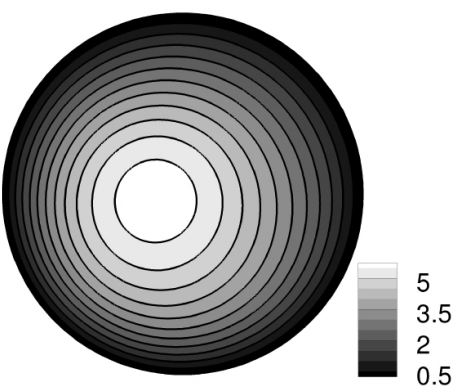

(a) $\mathrm{R}=0.1 \mathrm{D}$

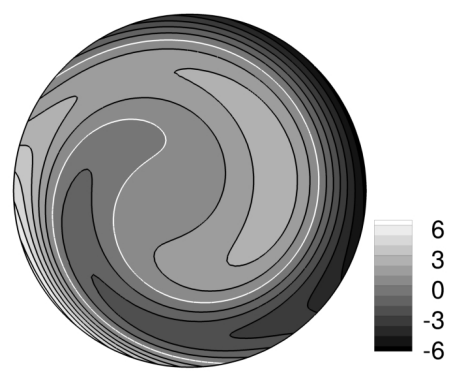

(d) $\mathrm{R}=0.1 \mathrm{D}$

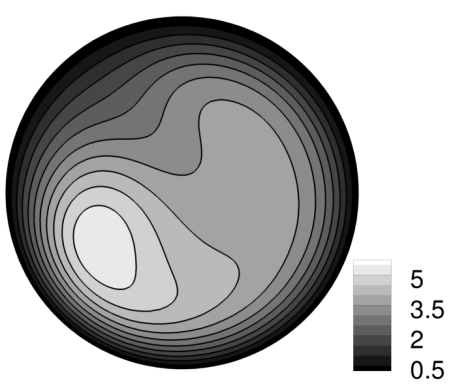

(b) $\mathrm{R}=0.3 \mathrm{D}$

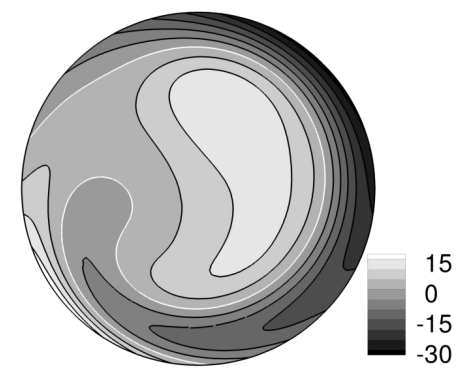

(e) $\mathrm{R}=0.3 \mathrm{D}$

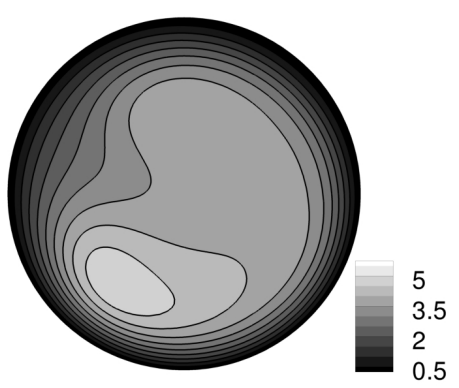

(c) $\mathrm{R}=0.5 \mathrm{D}$

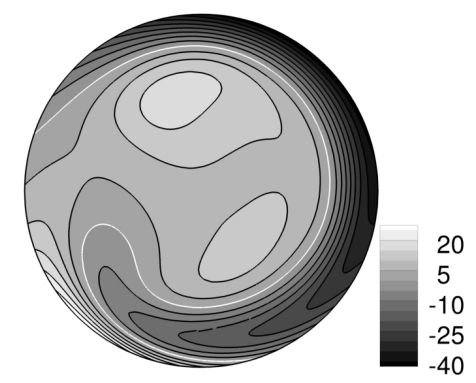

(f) $\mathrm{R}=0.5 \mathrm{D}$

Fig. 4. Axial velocity (upper) and axial vorticity (lower) contours at $z=6 D$. The zero contour of vorticity is shaded white.

convention allows comparison with results in the literature, and indeed our data is consistent with this work.

Figures 4(a) - 4(c) show contour plots of the axial velocity, where the axial direction is the z-axis in the Cartesian frame. For $R=0.1 D$ the axial velocity profile is close to that of Poiseuille flow, with only a small displacement of the peak velocity. As the radius, and hence curvature increases, the location of the peak velocity moves closer to the tube wall. The radial distance of the peak velocity, measured from the tube centre is plotted in Figure 5. The radial offset of the peak velocity increases rapidly with helical radius upto $R=0.25 D$, above this the rate of change of the offset is much smaller. Indeed, the data suggests that the value is reaching an asymptotic limit by $R=0.5 D$. 


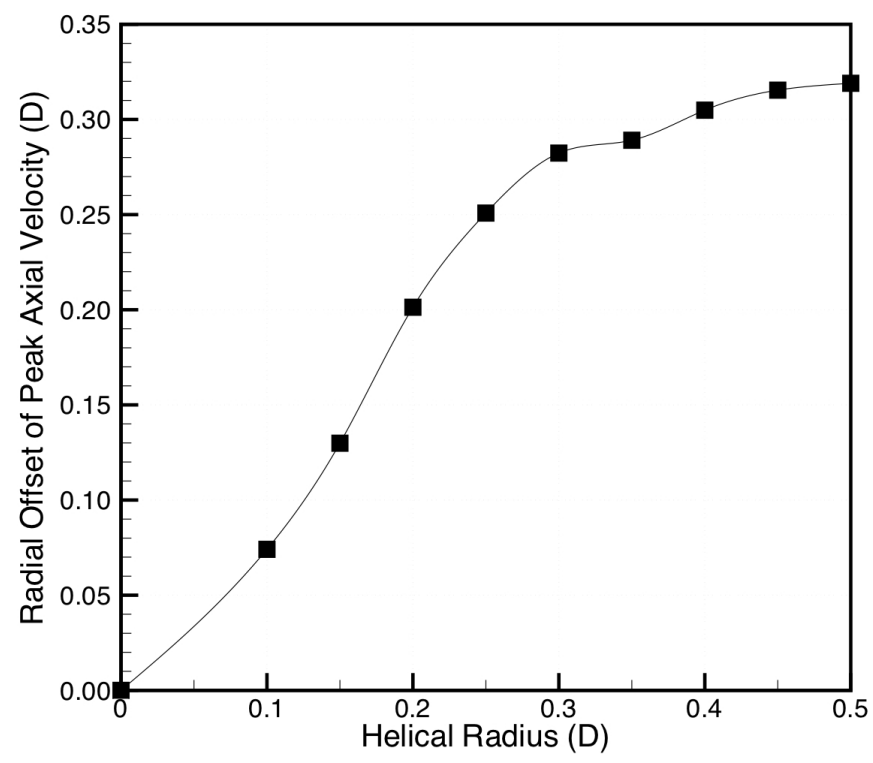

Fig. 5. Radial offset of peak axial velocity vs. helical radius in Cartesian co-ordinate system

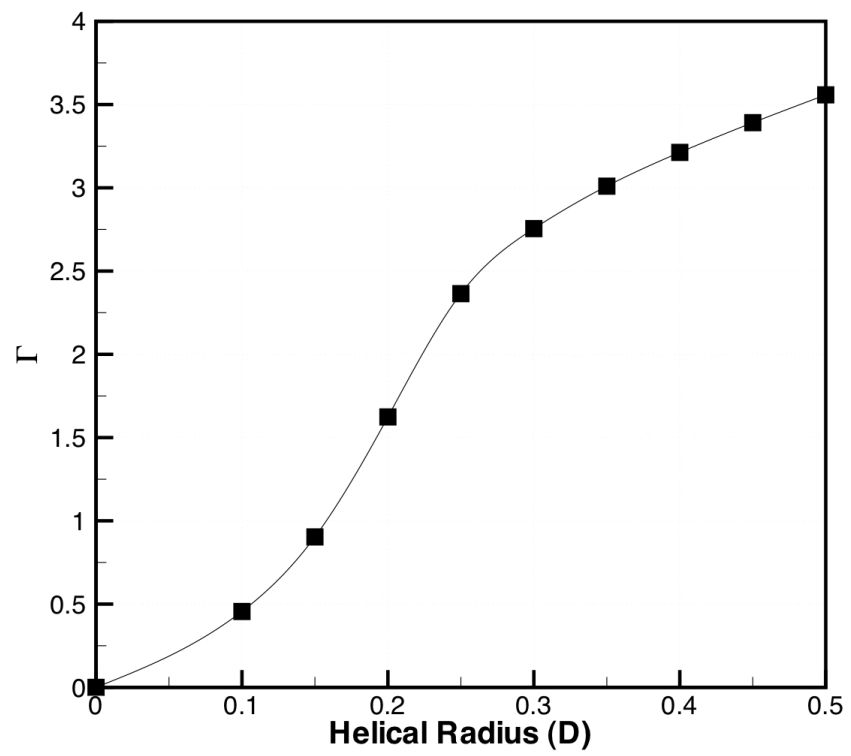

Fig. 6. Circulation, $\Gamma$, of dominant vortex vs. helical radius 
Figures 4(d) - 4(f) show the corresponding contour plots of the axial vorticity, $\omega_{z}$. A white contour line at $\omega_{z}=0$, highlights the regions of positive and negative vorticity, showing more clearly the vortical structure. For all cases there is a large region of positive vorticity, indicating that a single vortex dominates the flow, with a slight counter rotation near the wall. The existence of solutions of this nature has been reported previously by other researchers (Zabielski and Mestel, 1998) (Yamamoto et al., 2002). The contiguous region of positive vorticity is then integrated over the area to obtain the circulation $\Gamma$. The variation of $\Gamma$ with helical radius, shown in Figure 6, is similar in manner to that of the radial offset of peak axial velocity, upto a helical radius of $0.25 D$. For values of helical radius greater than this, the rate of increase in $\Gamma$ is reduced, although the peak axial velocity offset appears to asymptote. Clearly the radial location of the peak axial velocity is limited by the wall and its no-slip boundary condition. The circulation of the vortex, by contrast, is expected to increase with radius, since the curvature also increases, and with it the centrifugal force acting on the fluid.

\subsection{Mixing/Stirring}

In examining the mixing characteristics of these helical geometries we will first look at the qualitative behaviour of fluid particles, before quantifying the mixing using the entropy measure introduced in Section 2.4. An initial distribution of 15371 particles are seeded on a uniform grid, truncated by the circular cross-sectional boundary. The particles are tracked through the helical geometries for a total of five pitch lengths, a distance of $30 D$, a reasonable limit in terms of probable length constraints of clinical applications of a bypass 
graft. The inlet particle distribution is coloured by radius such that there are an equal number of grey and black particles. Besides their use in the entropy calculation, particle colouring indicates the degree of exchange of near wall particles with the core fluid. This is thought to be relevant to biological applications, where wall-transfer processes are implicated in disease initiation and progression (Nielsen, 1996).

Figure 7 shows the particle trajectory slices for the geometry $R=0.25 D$, starting with the initial distribution and then each subsequent integer pitch length. Comparing the particle distributions at successive downstream locations, the initial concentric colour distribution is seen to become increasingly mixed. Closer examination of the individual plots reveal common features of the particle distribution, such as the location of the dividing line between the two vortex-like regions. Although visible in all the plots, the finer structures within each of the two regions become better illuminated with each additional pitch length, since the particle colours are more uniformly distributed within the domain.

In Figure 8(a), comparison of the the mixing at a fixed downstream location of $30 D$ is shown for helical radii ranging from $0.1 D$ to $0.5 D$. From Figure 8 (a) it is clear that very little mixing is occurring for the geometry $R=0.1 D$. In fact, the section of black particles is merely rotated, with only a small amount of distortion, and crucially none of the near wall particles move to the core of the flow, even after five pitch lengths. This is in contrast to $R=0.25 D$, Figure 7(b) where even after one pitch length, the majority of particles initially seeded near the wall have moved to the core.

Viewing the individual plots of Figure 8 in sequence reveals the gradual tran- 


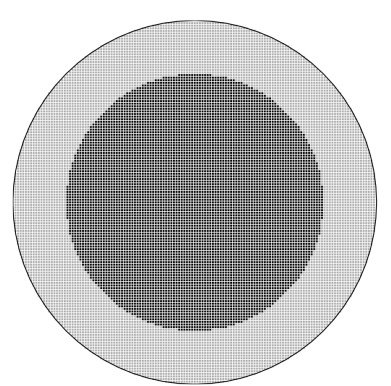

(a) Initial distribution

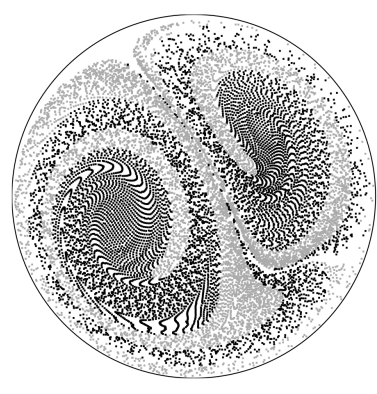

(d) $z=18 D$

(three pitches)

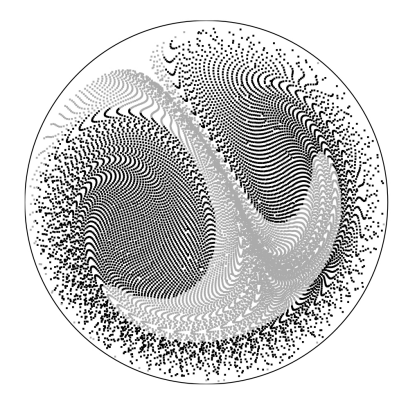

(b) $z=6 D$

(one pitch)

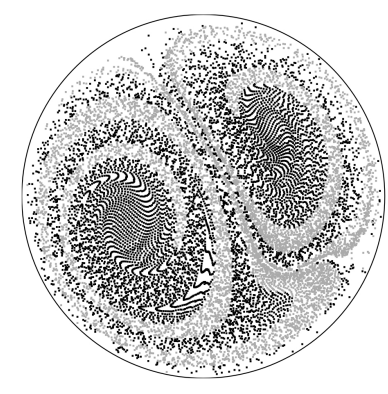

(e) $z=24 D$

(four pitches)

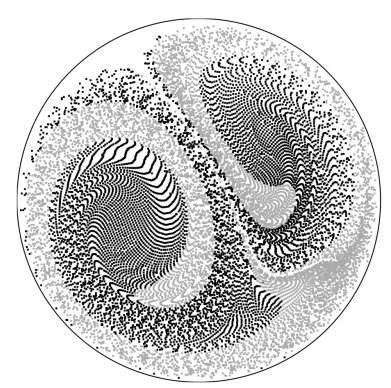

(c) $z=12 D$

(two pitches)

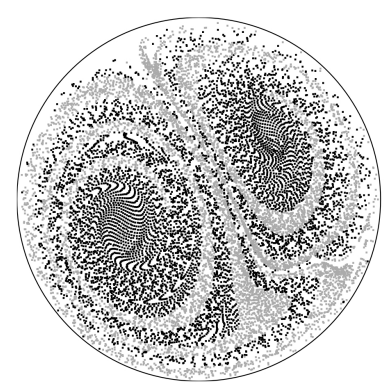

(f) $z=30 D$

(five pitches)

Fig. 7. Particle trajectory slices for $R=0.25 D$ at integer multiples of the pitch length

sition of the particle trajectories from a single vortex to a double vortex structure, despite Figures 4(d) - 4(f) indicating that there is only a single dominant vortical structure for all the radii investigated. This counter-intuitive phenomenon has also been reported by Yamamoto et al. (2002) in both their computational particle tracking and smoke visualisation results. They suggest that this feature occurs due to a combination of the secondary and axial velocities. This is essentially correct, and we provide a more complete explanation in the following. 


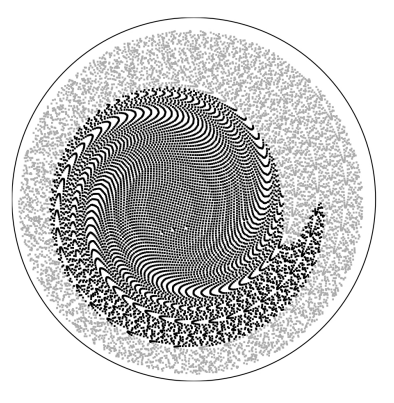

(a) $R=0.1 D$

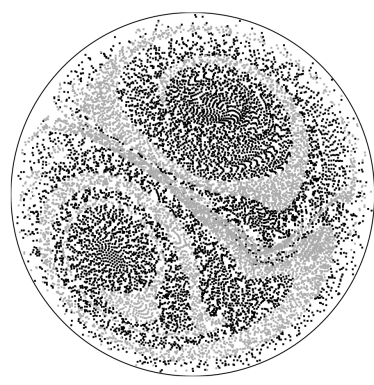

(d) $R=0.4 D$

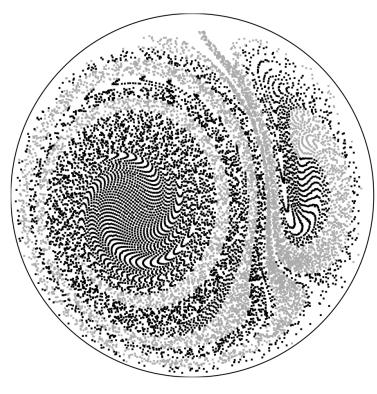

(b) $R=0.2 D$

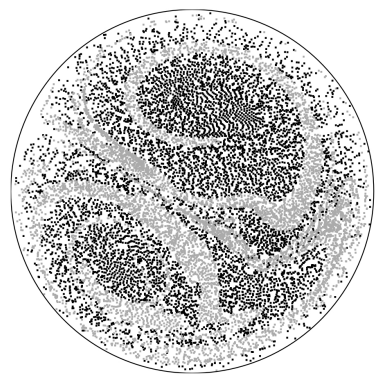

(e) $R=0.5 D$

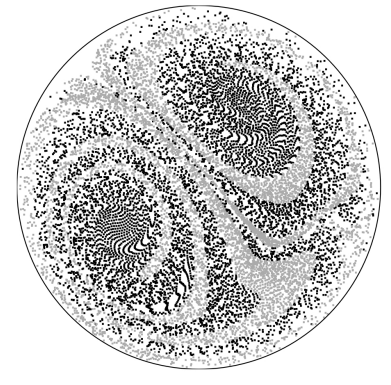

(c) $R=0.3 D$

Fig. 8. Particle trajectory slices at $z=30 D$ (five pitches) for varying helical radius

\subsection{In-plane particle trajectory}

Attempting to relate the in-plane mixing/stirring to the flowfield is complicated by the fact that the planar cross-section is both translating and rotating. Therefore to interpret the motion of particles we must remove the effects of the plane's translation and rotation. Note that the following arguments are only kinematic in nature, and are not concerned with the dynamics.

The velocity in the planar cross-section can be calculated by:

$$
\mathbf{V}_{\text {in-plane }}=\mathbf{V}_{\text {cartesian }}-\mathbf{V}_{\text {translation }}-\mathbf{V}_{\text {rotation }}
$$


and illustrated in Figure 9 for the case $R=0.25 D$. The translation and rotation velocities will now be considered in turn. For any particle convected by the flow with axial velocity $w$, the translational velocity of the cross-sectional plane can be derived by differentiating the helical centreline equations with respect to time. Writing $w=d z / d t$, the corresponding rate of change of position of the helix centreline is given by:

$$
\begin{aligned}
& \dot{x}_{\text {translation }}=-(w R / c) \sin (z / c) \\
& \dot{y}_{\text {translation }}=(w R / c) \cos (z / c)
\end{aligned}
$$

For example, at $z=6, \dot{x}_{\text {translation }}=0$, and $\dot{y}_{\text {translation }}=w R / c$.

Figure 9(b) shows the streamtraces for the in-plane velocities in the Cartesian frame, overlaid on a contour plot showing the magnitude of these velocity components for comparison with the axial velocity. The streamtraces show that the flow is not confined to the plane, due to the movement of the crosssection along the helix, and are thus sensible in the context of a $3-D$ geometry. Removing the translation velocity produces the velocity field in Figure 9(c). This in-plane flow is now confined to the cross-section, forming one large vortex, and a small counter rotating vortex near the wall.

As we have already observed, the flow profile in a helical tube also rotates in a self similar method along the length of the helix. Given a pitch length of $c$, the apparent rate of rotation of a point at a distance $r$ from the tube centre, travelling at an axial velocity $w$ is given by:

$$
\dot{\theta}_{\text {rotation }}=w / c
$$




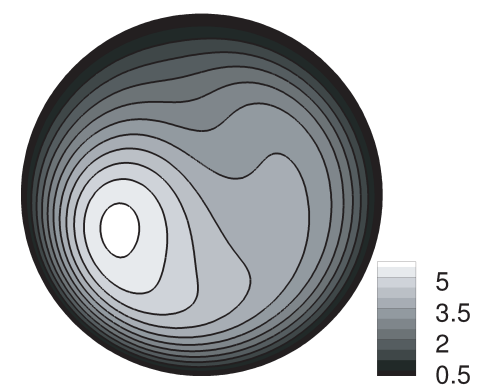

(a) $\mathbf{V}_{\text {cartesian }}, w$

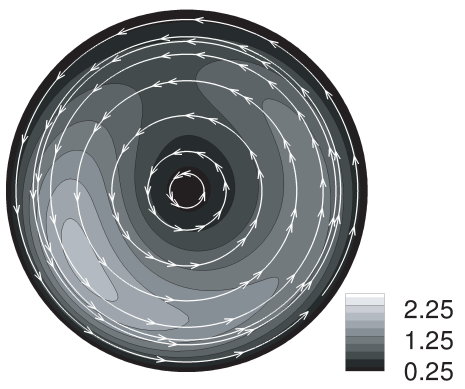

(d) $\mathbf{V}_{\text {rotation }}$

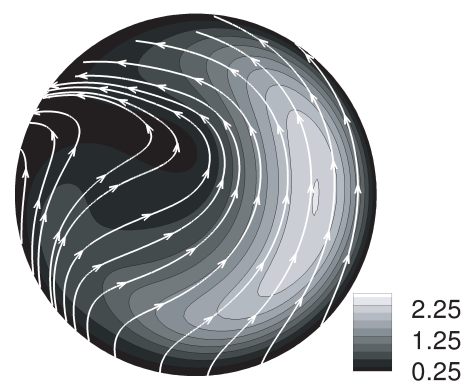

(b) $\mathbf{V}_{\text {cartesian }}$

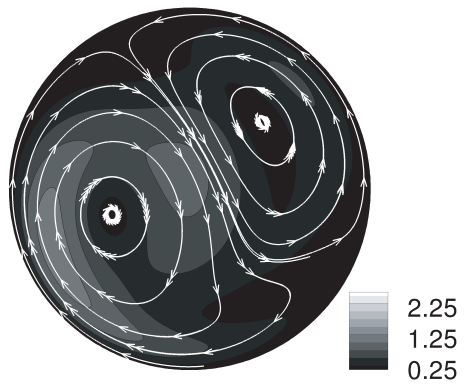

(e) $\mathbf{V}_{\text {in-plane }}$

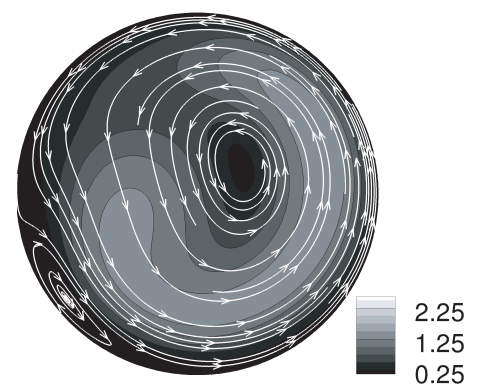

(c) $\mathbf{V}_{\text {cartesian }}$

$\mathbf{V}_{\text {translation }}$

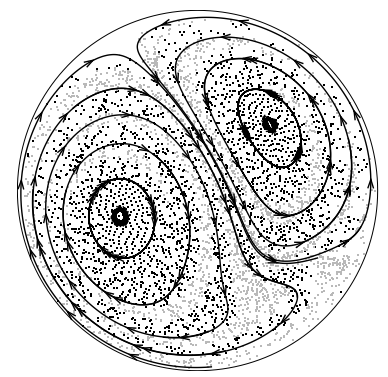

(f) $\mathbf{V}_{\text {in-plane }}$

Fig. 9. In-plane velocity transformations to obtain particle trajectories and therefore the tangential velocity is:

$$
V_{\text {rotation }}=w r / c
$$

The sign of rotation of this velocity is the same as that of the Cartesian vortex in the flow. Therefore when the rotating reference frame velocity is subtracted from the Cartesian in-plane flow, the resulting in-plane flow field depends on the relative strengths of these two contributions, and their distribution of velocity magnitude within the plane.

Figure $9(\mathrm{~d})$ shows the vortical structure induced by the rotation of the cross section. Although its core is at the centre of the tube, the highest velocities occur near the peak of the axial velocity, as comparison with Figure 9(a) 
indicates. Figure 9(e) shows the velocity streamtraces for the in-plane reference frame, overlaid on contours of the velocity magnitude. The average magnitude of the in-plane velocity is roughly half of that in the Cartesian frame, and almost seven times smaller than the mean axial velocity, which means that an average particle will move from one side of the plane, to the other, as it travels one pitch length of the helix. Figure 9(f) shows these same velocity streamtraces, overlaid on the particle trajectory slice for the same case. The correspondence between the two is excellent; the location of the vortex cores coincide, as does the angle of the dividing line between the vortical structures, and clearly supports the reference frame explanation of the particle mixing.

\subsection{Relationship between velocity field and mixing}

We now outline how the changes in location of peak axial velocity and circulation affect the in-plane stream-trace plots for different values of $R$. In the case $R=0.1 D$ the vortex in the flow is weak, and due to the small curvature of the tube, the axial velocity profile resembles a slightly perturbed Poiseuille flow. Therefore, when the axial velocity is multiplied by radial position, the rotational velocity produced will be relatively uniformly distributed. When subtracted from the physical vortex the resulting flow is a vortex in the opposite direction to the Cartesian one, and hence the particles are merely rotated with almost no mixing occurring.

For the case $R=0.3 D$ the circulation of the Cartesian vortex is almost 6 times larger than for $R=0.1 D$. Therefore in the positive $x$ and $y$ quadrant this vortex dominates the one from the rotational reference frame, but due to the large radial shift of the peak axial velocity towards the lower-left wall, 
the peak velocities in this vortex are larger than previously, and therefore this vortex dominates the negative $x$ and $y$ quadrant. In this way two vortices of opposite rotation are created in the in-plane reference frame. The situation is similar for $R=0.5$ except that the Cartesian vortex is slightly stronger and therefore the second in-plane vortex is itself stronger, and consequently the stream-traces occupy more of the cross-section.

Although the structure of the apparent vortex which arises from the rotation of the reference frame is the same for all of the geometries considered, the distribution of the velocity changes, due to the change in axial velocity profile. This means that the summation of both vortices also changes with helical radius.

To quantify the mixing behaviour, we now employ the concept of information entropy. Figure 10 shows the normalised entropy increase, $\kappa$, plotted against helical radius. It is clear that entropy increases, and therefore mixing, for all cases, apart from $R=0.1 D$. Furthermore, Figure 10 shows that there is a sharp increase in mixing upto and including $R=0.25 D$, beyond which there is only a small increase in $\kappa$ with increasing helical radius at five pitch lengths. The trend exhibited by this data is very similar to that for $\Gamma$ and the location of peak axial velocity. The particle distributions and the reference frame explanation suggests that mixing increases with the size of the apparent second vortex structure in the particle trajectories. Indeed it is this apparent structure that generates the particle mixing, as it increases the length of the interface between the particles within the plane.

With increasing radius, $\Gamma$ increases, and hence the strength second apparent vortex in the in-plane reference frame increases, at the expense of the first 
apparent vortex. This explains why the gradient of $\kappa$ for $R>0.25 D$ is smaller compared to the gradient for $R<0.25 D$, than is the case for $\Gamma$.

If the radius of the helix were increased far beyond $R=0.5 D$, forming a toroidal-like configuration, the in-plane velocity field would be two vortices, as reported in previous studies. For these geometries, where our Cartesian formulation of the problem is no longer appropriate, the strength of the vortex induced by the rotation of the reference frame reduces with helical radius, due to the increased arc length. Therefore, after substraction from the in-plane velocity field, it is likely that the two vortices will remain, although with altered strengths. Therefore, the mixing pattern of the flow will be similar to that reported here. However, the smaller centrifugal forces implies weaker vortices, and therefore mixing will likely decrease with radius for these cases.

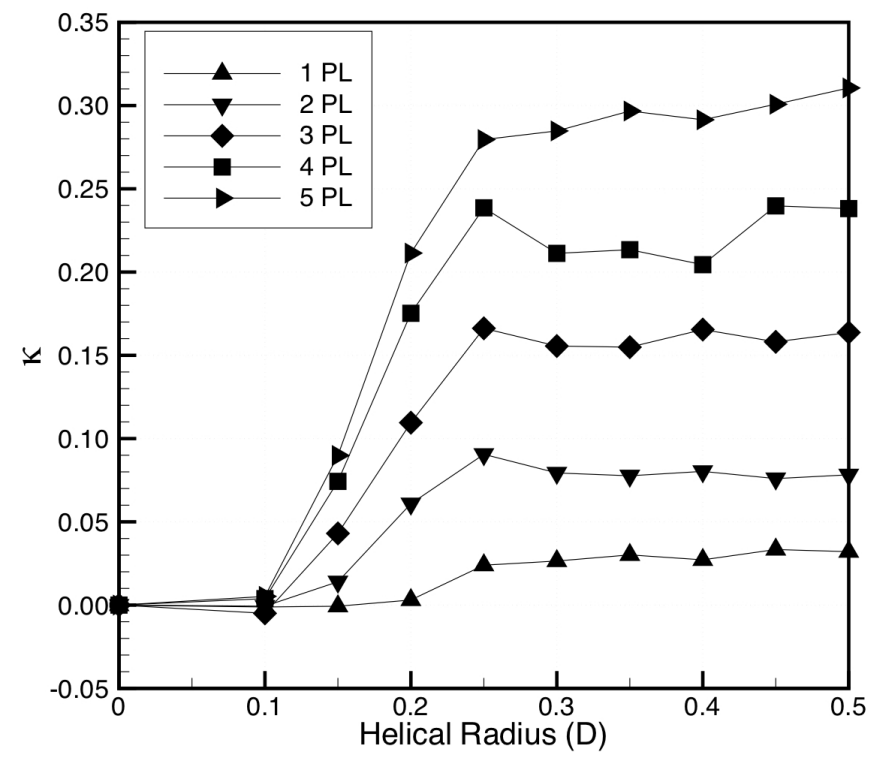

Fig. 10. Relative entropy, $\kappa$, vs. helical radius 


\subsection{Mixing at higher Reynolds number}

In order to provide some indication of the mixing expected for operating conditions appropriate for an $\mathrm{A}-\mathrm{V}$ shunt, flow solutions have been obtained for a selection of geometries at higher Reynolds numbers. Figure 11 shows streamtraces of $V_{\text {in-plane }}$ for the cases $R=0.1 D, 0.25 D \& 0.4 D$, at Reynolds numbers of approximately 250 and 500. It is clear that there is only a slight difference in structure between the two Reynolds numbers, although the magnitudes are obviously different. The second (upper) vortex which forms with increasing $R$, occupies slightly more of the cross-section at $R e=500$, the difference being smaller for $R=0.4 D$. Extrapolating these results to $R e \approx 900-1000$, it is predicted that the helical radius at which "sufficient" mixing is generated will be slightly smaller than for $R e=250$, but likely no smaller than $R=0.2 D$.

These results come with the caveat that the precise Reynolds number at which flow transitions to turbulence within helical geometries is not properly characterised as a function of geometric parameters. One study that attempted to understand this phenomenon (Yamamoto et al., 1998) investigated geometries similar to those considered in this study, with comparative pitch lengths, though not for values of $R$ as small as here. The results suggest that transition occurs at values of Re smaller than for a straight pipe $\mathrm{O}(2000)$, possibly as low as $R e=800$, depending on the geometry. An interesting property of the flow is that torsion is initially destabilising, but beyond a certain point, increasing torsion will increase the critical transition Reynolds number. If the flow does transition to turbulence then we concede that the results presented here will no longer apply. It is clear that a careful analysis of the stability of such flows is needed, but this is beyond the scope of the present investigation. 


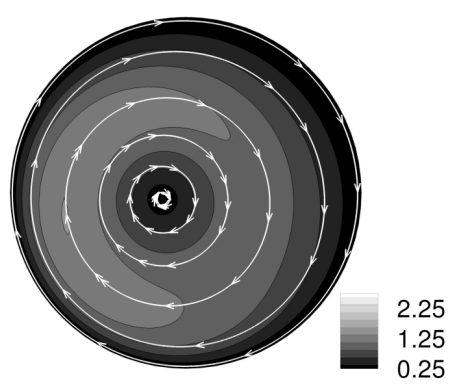

(a) $R=0.1 D, R e=$ 250

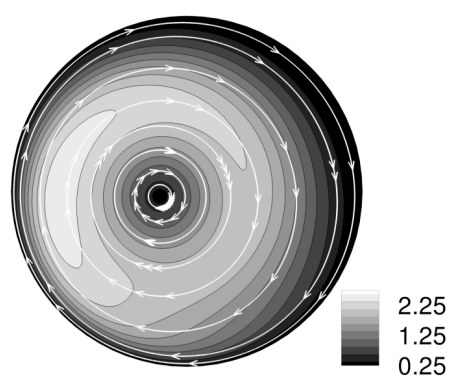

(d) $R=0.1 D, R e=$ 500

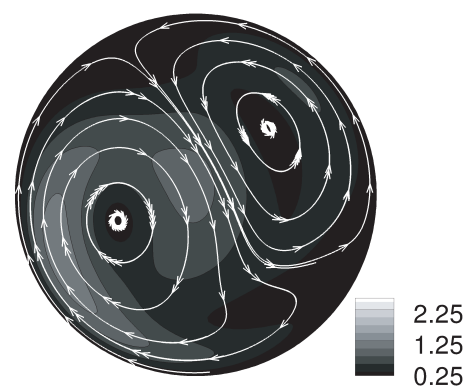

(b) $R=0.25 D, R e=$ 250

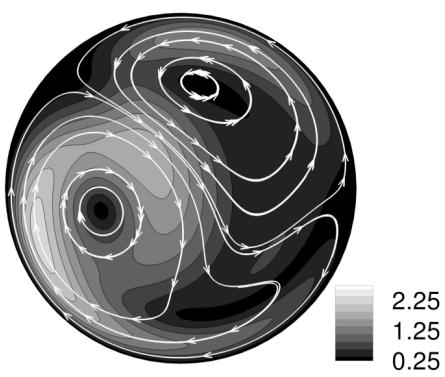

(e) $R=0.25 D, R e=$ 500

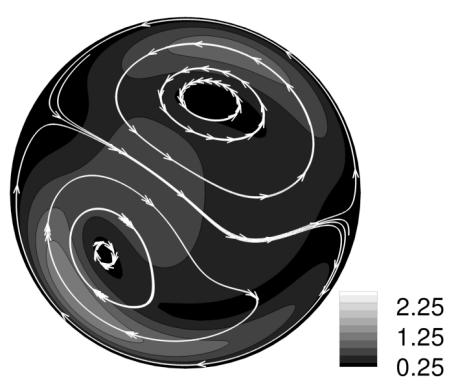

(c) $R=0.4 D, R e=$

250

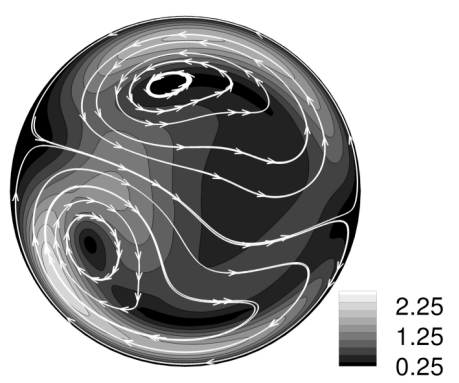

(f) $R=0.4 D, R e=$ 500

Fig. 11. Streamtraces of $V_{\text {in-plane }}$ for $R e=250$ and 500 for several helical geometries

\subsection{Particle residence times}

Thus far we have examined only the in-plane mixing, however dispersion of the particles in the axial direction is also of relevance. For the particles tracked in Section 3.2 the time taken to travel through one pitch length was recorded. The mean and standard deviation of the residence times are shown in Figure 12. It is clear that both quantities vary in a similar fashion, and since a lower mean residence time is desirable, the trend is the same as for $\kappa$ in Figure 10. The lower average residence time with increasing radius is due to the greater in-plane mixing, which means that more of the particles are brought into the core of the flow, and thus experience the high axial velocities there. The smaller 
standard deviation, by definition, means that the residence times are closer to the mean value, and hence the residence time distribution is more uniform for the larger radii. In the preliminary study by Caro et al simple dye injection experiments were performed for two U-bend configurations; one constructed using conventional cylindrical tube, and the other a helical tube with radius $0.5 D$ (Caro et al., 2005). The axial dispersion was lower for the helical tube, as was the retention of dye near the inner wall of the bend. Though we have only considered a straight section, those results are in accordance with our findings.

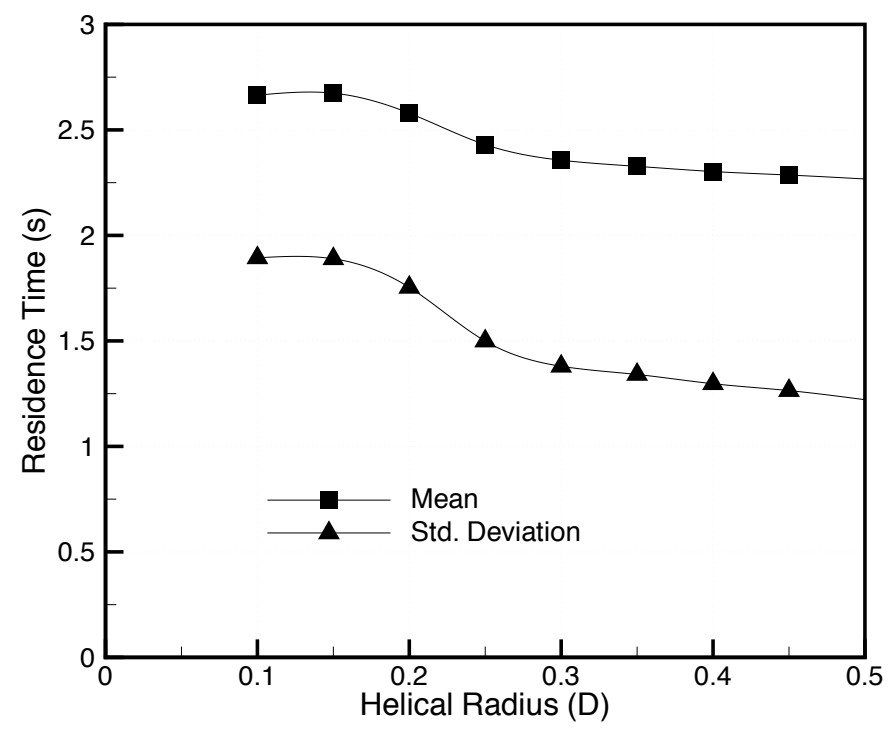

Fig. 12. Statistics of the particle residence times distributions for varying helical radius

It is suspected that the effect of fluid mixing on residence time distributions provides the link to reduced thrombus formation. Consider the activation parameter defined by Ramstack et al. (1979), which is the product of shear rate and exposure time. They show that if this parameter exceeds 1000, proco- 
agulant platelet factor 3 (FP3) is released, thus enabling the formation of a thrombus. In a Poiseuille flow the shortest axial distances sufficient to cause FP3 release occur in the near wall region, creating the ideal conditions for a thrombus to form on the tube's surface. In the helical geometry, the more uniform residence time distribution implies that extremes of shear exposure time are reduced, likely reducing the number of activated platelets. Additionally, the mixing of particle between the near wall region and core of the flow, facilitated by the double-vortex structure, helps prevent activated platelets from residing near the wall and ultimately accumulating.

A key result from quantifying the degree of mixing, is that there is a relatively small difference in the mixing performance between the cases $R=0.25 D$ and $R=0.5 D$. Therefore if enhanced in-plane mixing, as we have defined and measured it, is responsible for the apparent improved performance of the SMAHT A-V shunts used by Caro et al. (2005), it would appear that a helical geometry with $R=0.25 D$ should be just as effective at preventing thrombogenic occlusion, as the $R=0.5 D$ geometry. A further consideration when selecting a geometry for medical applications, is the magnitude of the pressure drop across one pitch length of the helix. As the helical radius increases, pressure losses will arise from both the greater total arc length of the helix, and the energy required to drive the vortical structure. Figure 13 shows the variation of this pressure drop with helical radius, indicating that the pressure loss for $R=0.5 D$ is approximately 1.6 times larger than for $R=0.25$, but without a corresponding increase in the degree of mixing. 


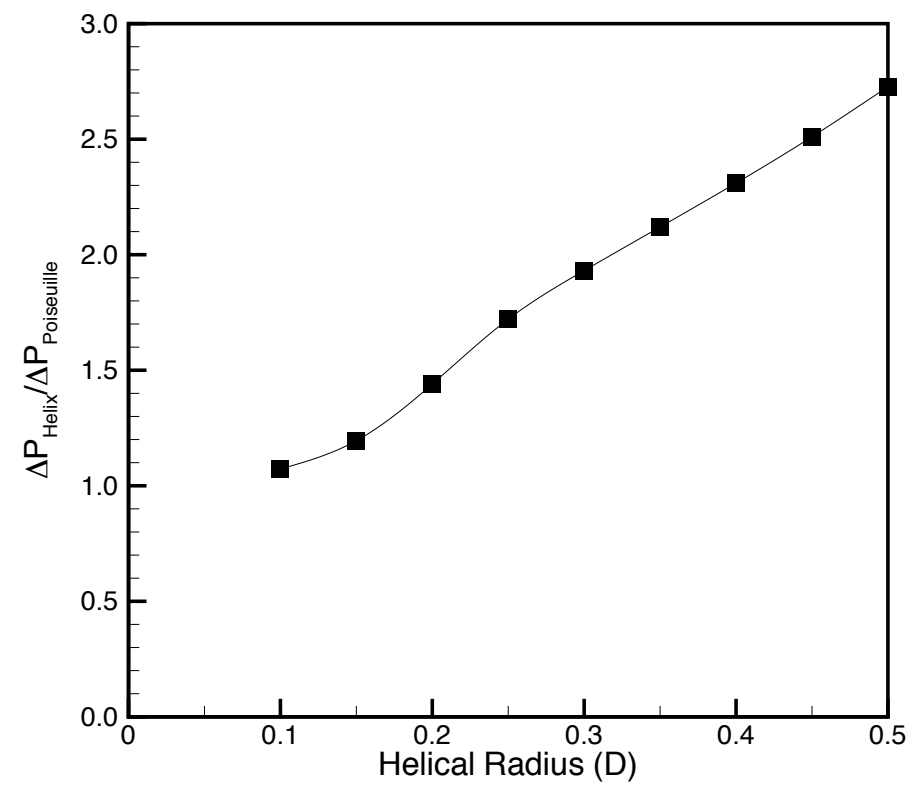

Fig. 13. Pressure drop along one pitch vs. helical radius

\subsection{Implications of in vivo conditions for helical prostheses}

The focus of this study has been to understand the fundamental process of mixing in helical geometries, hitherto a problem not fully resolved in the fluid mechanics literature. When used as a prosthetic device, several in vivo conditions may have an effect on the flow structures, and hence the mixing. Here we comment on the likely significance of these additional factors, and their implications for the conclusions drawn regarding optimal geometries for medical applications.

The first effect, and the most significant, is the presence of an additional curvature in part, or all, of the shunt geometry, arising due to the loop configuration commonly used to connect the artery to the vein. To a first approximation, the flow in a helical tube formed on a curved centreline, can be taken as the superposition of symmetric counter-rotating Dean vortices, and the single- 
vortex swirling flow of a helical tube. Since, the velocity field in the helical pipe varies only slightly with increasing radius for $R>0.25$, this means the resultant flow in a curved helical tube will not vary significantly in this parameter range. Therefore, despite the precise mixing behaviour differing from the straight helical case, the ranking of geometries from a mixing perspective should not be altered by the presence of additional curvature.

Whilst the additional curvature is relevant to the A-V shunt application, for the cases of a bypass graft and arterial stent, the additional curvature will be small, and negligible in its effect. Related to this point, an interesting structural property of helical tubes is their improved resistance to kinking when subjected to bending moments, compared to conventional straight cylindrical tubes. This property helps to guarantee the geometry of the graft in vivo, and prevents extreme curvatures, due to a kink, that would more easily cause the flow to separate. Related to this, a recent study by Coppola and Caro (2008) that investigated a helical geometry formed on a curved centreline showed that even at $R e=600$ the flow remained attached throughout the bend, so that even though the mixing behaviour may be altered, the additional curvature is unlikely to have a pathological effect.

The second point to consider is the possibility of pressure-compliance mismatches at the junctions of the shunt. This is a problem associated with grafts in general, and it has been hypothesised that this mismatch might play a role in the development of intimal hyperplasia near the suture line (Leuprecht et al., 2002). Whilst the cross-plane pressure gradient that exists in the helical pipe means this may be slightly different to the straight pipe case, it is not thought to be significant, and nor would it have such a direct bearing on the rate of development of thrombosis within the graft itself. 


\section{Conclusions}

The results presented here promote an explanation of the mechanics of scalar particle mixing in helical tubes. The dependence of mixing effectiveness on the radial offset of peak axial velocity and strength of the vortical structures are determined. If further studies of mixing in helical tubes are to be performed, then a good estimate of the mixing can be made using these quantities, without the need for $3-D$ particle tracking analysis; a large saving in computational effort, enabling a larger parameter space to be examined. Quantifying this mixing using an information entropy method, and particle residence times shows that beyond $R=0.25 D$ only a small increase in mixing occurs with increasing helical radius, which may be important for clinical applications.

\section{Acknowledgements}

The authors would like to acknowledge the EPSRC for funding this research.

\section{References}

Bryan, A. J., Angelini, G. D., 1994. The biology of saphenous vein graft occlusion: etiology and strategies for prevention. Current opinion in cardiology $9(6)$.

Butler, J. P., Tsuda, A., 1997. Effect of convective stretching and folding on aerosol mixing deep in the lung, assessed by approximate entropy. J Appl Physiol 83, 800-809.

Caro, C. G., Cheshire, N. J., Watkins, N., 2005. Preliminary comparative 
study of small amplitude helical and conventional eptfe arteriovenous shunts in pigs. Journal of the Royal Society interface 2, 261-266.

Caro, C. G., Doorly, D. J., Tarnawski, M., Scott, K. T., Long, Q., Dumoulin, C. L., 1996. Non-planar curvature and branching of arteries and non-planartype flow. Proceedings: Mathematical, Physical and Engineering Sciences 452 (1944), 185-197.

Coppola, G., Caro, C., September 2008. Oxygen mass transfer in a model three-dimensional artery. Journal of the Royal Society Interface 5 (26), $1067-1075$.

Coppola, G., Sherwin, S. J., Peiro, J., 2001. Non-linear particle tracking for high-order elements. Journal of Computational Physics 172.

Darekar, R. M., Sherwin, S. J., 2001. Flow past a square-section cylinder with a wavy stagnation face. Journal of Fluid Mechanics 426, 263-295.

Doorly, D. J., 1999. Flow transport in arteries. In: Sajjadi, S. G., Nash, G. B., Rampling, M. W. (Eds.), Cardiovascular Flow Modelling And Measurement With Application To Clinical Medicine. Oxford University Press, pp. 67-81.

Doorly, D. J., Sherwin, S. J., Franke, P. T., Peiro, J., 2002. Vortical flow structure identification and flow transport in arteries. Computer Methods in Biomechanics and Biomechanical Engineering 5 (3), 261-275.

Evangelinos, C., 1999. Parallel spectral/hp methods and simulations of flow/structure interactions. Ph.D. thesis, Brown University.

Friedman, M. H., 1993. Arteriosclerosis research using vascular flow models: from 2-d branches to compliant replicas. Journal of biomechanical engineering 115 (4B), 595-601.

Germano, M., 1981. On the effect of torsion on a helical pipe flow. Journal of Fluid Mechanics 125, 1-8.

Huijbregts, H. J. T. A. M., Blankestijn, P. J., Caro, C. G., Cheshire, N. J. W., 
Hoedt, M. T. C., Tutein Nolthenius, R. P., Moll, F. L., 2007. A helical ptfe arteriovenous access graft to swirl flow across the distal anastomosis: Results of a preliminary clinical study. European Journal of Vascular and Endovascular Surgery 33 (4), 472-475.

Kang, T. G., Kwon, T. H., 2004. Colored particle tracking method for mixing analysis of chaotic micromixers. Journal of Micromechanics and Microengineering $14,891$.

Khakhar, D. V., 1986. Analysis of chaotic mixing in two model systems. Journal of fluid mechanics 172 .

Koberg, W. H., 2008. Turbulence control for drag reduction with active wall deformation. Ph.D. thesis, Imperial College London.

Krasnopolskaya, T. S., Meleshko, V. V., Peters, G. W. M., Meijer, H. E. H., 1999. Mixing in stokes flow in an annular wedge cavity. European journal of mechanics. B, Fluids 18 (5).

Leuprecht, A., Perktold, K., Prosi, M., Berk, T., Trubel, W., Schima, H., 2002. Numerical study of hemodynamics and wall mechanics in distal end-to-side anastomoses of bypass grafts. Journal of Biomechanics 35, 225-236.

Loth, F., Fischer, P. F., Bassiouny, H. S., January 2008. Blood flow in end-toside anastomoses. Annual Review of Fluid Mechanics 40, 367-393.

Newman, D., 1996. A computational study of fluid/structure interactions: flow-induced vibrations of a flexible cable. Ph.D. thesis, Princeton University.

Nielsen, L. B., 1996. Transfer of low density lipoprotein into the arterial wall and risk of atherosclerosis;. Atherosclerosis 123 (1-2), 1-15.

Ramstack, J. M., Zuckerman, L., Mockros, L. F., 1979. Shear-induced activation of platelets. Journal of Biomechanics 12 (2), 113-25.

Shannon, C. E., 1948. A mathematical theory of communication. Bell System 
Technical Journal 27, 379-423.

Sherwin, S. J., Karnaidakis, G. E., 2005. Spectral/hp Element Methods for Computational Fluid Dynamics. Oxford Science Publications.

Sherwin, S. J., Shah, O., Doorly, D. J., Peiro, J., Papaharilaou, Y., Watkins, N., Caro, C. G., Dumoulin, C. L., 2000. The influence of out-of-plane geometry on the flow within a distal end-to-side anastomsis. ASME J. Biomech $122,86-95$.

Wang, C. Y., 1981. On the low-reynolds-number flow in a helical pipe. Journal of Fluid Mechanics 108, 185-194.

Wang, W., Manas-Zloczower, I., Kaufman, M., 2003. Entropic characterization of distributive mixing in polymer processing equipment. AIChE Journal 49, $1637-1644$.

Wang, W., Manas-Zloczower, I., Kaufman, M., 2005a. Entropy time evolution in a twin-flight single-screw extruder and its relationship to chaos. Chemical Engineering Communications 192, 405-423.

Wang, W., Manas-Zloczower, I., Kaufman, M., 2005b. Influence of initial conditions on distributive mixing in a twin-flight single-screw extruder. Chemical Engineering Communications 192, 749-757.

Yamamoto, K., Aribowo, A., Hayamizu, Y., Hirose, T., Kawahara, K., 2002. Visualization of the flow in a helical pipe. Fluid Dynamics Research 30, $251-267$.

Yamamoto, K., Yanase, S., Jiang, R., 1998. Stability of the flow in a helical tube. Fluid Dynamics Research 22, 153-170.

Zabielski, L., Mestel, A. J., 1998. Steady flow in a helically symmetric pipe. Journal of Fluid Mechanics 370, 297-320. 